\title{
THE SURGERY OF RHEUMATIC HEART DISEASE
}

\author{
By J. R. BelChER, M.S., F.R.C.S.
}

Rheumatic heart disease as it affects the cardiac valves may give rise to stenosis or incompetence. Although these processes may affect any valve, the lesions in the left side are by far the most common and of the most clinical significance.

Hitherto the surgical attack on the cardiac valves has been mostly confined to the stenotic lesions, although attempts have already been made to deal with valves made incompetent by disease or by operation aimed to relieve stenosis. Murray (1950) after resection of part of a rigid mitral valve created a new one by the use of a vein threaded inside out on a tendon, which was placed in position in the ventricle under direct vision through a cardioscope in such a way as to create a ball valve over the artificial defect.

So far attempts to relieve aortic stenosis have been a little disappointing although successful cases have now been reported by Bailey (1950) who after many and various experiments succeeded in dilating a stenosed aortic valve from above through the right carotid artery.

There remains mitral stenosis and it is the treatment of this lesion which will be dealt with here, as the operation of ' commissurotomy' now carries a low morbidity and mortality, is often spectacularly successful and seldom fails to afford a considerable measure of relief to grossly disabled patients.

\section{Historical}

Brunton in 1902 seems to have been the first to moot the idea that the treatment of mitral stenosis might be brought into the realms of surgery, but it was not until 1923 that a patient suffering from this condition was operated upon. In that year Cutler successfully divided a valve and later performed several similar operations.

In I925 Souttar successfully performed an operation very similar in conception to the modern ' commissurotomy.' Thus from the beginning the two techniques of 'valvuloplasty' and 'commissurotomy' were practised.

At the start it was felt that any operation which was directed at enlarging the mitral orifice must necessarily lead to increased incompetence, and where actual division of the valve has been carried out at a point anywhere other than at the line of fusion (commissure), this has almost always been true. It was also soon realized that any deliberate or accidental division of the antero-medial, or aortic cusp, led to severe regurgitation and was rapidly fatal, but that the regurgitation caused by division of the smaller postero-lateral cusp was well borne and might indeed be negligible. The operation of 'valvuloplasty' as performed" by Cutler and later by Harken (1948) was based on these theoretical assumptions, and regurgitation was accepted as inevitable. The conception of ' commissurotomy' is different in principle.

Bailey (1949) believed that if the lines of fusion between the valve cusps $s^{z}$ could be broken down it might be possible to restore almost normal function without any incompetence. On this assumption he developed the now widely accepted operation of ' commissurotomy.' Despite the initial fatalities he was able to report a successful case in 1948 , and since then has carried out the operation on more than 800 occasions. In this country the first successful series since that of Souttar was published by Brock in $195^{\circ}$.

At the same time various other methods for relieving patients with mitral stenosis were developed. In principle these consisted of, either an attempt to relieve the associated pulmonary hypertension and so to alleviate many of the ill effects of the stenosis, or of an attempt to by-pass the valve itself.

Under the first heading Sweet in America and D'Allains in France undertook the anastomosis of a pulmonary to a systemic vein (the azygos and inferior pulmonary veins on the right side were used) and considerable subjective relief was achieved in patients subjected to this procedure. At the same time Harken attempted to overcome the ill effect of the back pressure in the pulmonary veins by the creation of an inter-atrial septal defect and achieved some relief by this means.

The attempts made to by-pass the stenotic valve have so far been experimental and unsuccessful, and as the more direct method of attack on the valve has so much to recommend it, it seems 
likely that there will be few further attempts to produce such a by-pass.

Hitherto all the operations performed on the mitral valve have been essentially 'blind ' as there has been considerable difficulty in developing an adequate cardioscope which is at the same time small enough to introduce through the auricular appendage. Butterworth (195I), however, has recently devised a perspex instrument, and although he does not record its successful use in man, it seems likely that he will very shortly do so and that the whole operation will be revolutionized.

\section{Anatomy}

The normal mitral valve has two parts, a large antero-medial cusp and a smaller postero-lateral one. Thus the orifice runs in an oblique direction postero-medially. The anterior or aortic cusp is the more important of the two and its function during ventricular systole is to direct the flow of blood into the aortic orifice.

The papillary muscles arising in the ventricle are inserted near to, but not at, the margin of the valves so that during ventricular systole the cusps billow up like sails in the wind and are opposed over quite a wide area; any lesion which leads to shortening of the tendons of these muscles must necessarily damage this mechanism.

\section{Pathology}

The rheumatic process affects every part of the heart, but its most damaging lesions occur in the valves, on their edges and in the chordae tendinaea.

When the active vulvulitis has subsided the mitral valve cusps are fused along the greater part of their length to form the ' commissures' described by Bailey. There may be calcification and vegetations along these 'commissures,' but the remainder of the valve may be almost normal, and if the chordae tendinaea have not become shortened the Type I valvular stenosis of Harken is present. If the inflammatory process has seriously damaged the whole valve and has involved the chordae tendinaea and the papillary muscles, the valve becomes fixed and funnel shaped and the actual tendons which are inserted into the different cusps may become fused to each other. This is the state of affairs in the much less common Type 2 stenosis of Harken and incompetence is more likely in this type of case.

As Brock pointed out at the meeting of the Association of Thoracic Surgeons in 1951, the ' commissures' are not fused with equal firmness throughout their length. The degree of fusion corresponds closely to the number of chordae inserted at any point on the valve edge; thus the medial third of the valves are seldom densely adherent and can be separated easily with the finger. Similarly the lateral third often splits easily and it is only in the middle third wherø tendinous insertion is thickest and where fusion of the chordae may have taken place that a cutting instrument may be required to split the 'coms? missure' in the correct direction.

\section{Selection of Cases for Operation}

In this field opinion is at present altering almos from day to day and several conditions which werê regarded as absolute contra-indications a year ago are now almost disregarded in pre-operative assessment.

Two principles seem important, first the opera tion is not curative and is aimed only at the alteration of the mechanics of the valve; secondly the condition of the cardiac muscle has to be taker into consideration.

The indication for operation is not the merêr presence of mitral stenosis for, as Bourne (1952) points out, many patients who have all the clinicaP signs of mitral stenosis have virtually no symptoms and all attempts at classification of patients have included one group who had no disability. Theres have been two different approaches to the problem of classification; those based on physical signs aod data discovered during investigation (Harke्ñ, I950), and those based on the history given by patient-subjective signs.

The latter method is preferable. It is notorious difficult to assess a patient's degree of disabilityo from direct questioning and it is, therefore essential that some simple exercise' test, such as, climbing stairs, should be employed in all cases (Somerville, 1952).

Extensive catheterization investigations have $\overrightarrow{\overrightarrow{0}}$ been done and will undoubtedly give useful in 3 formation as to the severity of the disease and likely outcome of operation. The actual suitability for operation cannot yet be reliably decided by this? method. Although pre- and post-operativecatheterization may be helpful, these procedures? are by no means essential in the course of treat-i ment of mitral stenosis. Bailey (1950) divides his cases into five classes: Asymptomatic; static incapácitating; progressive incapacitating ; terminal incapacitating; irretrievable; and confines his operation to the middle three groups.

The contra-indications are decreasing but a few remain. The possibility of reactivation of the rheumatic process is always present in patients below the age of 20 and therefore operation is $\omega$ usually contra-indicated in a patient below this age. The upper age limit has now reached the fifties. Clearly the presence of active rheumatice disease is an absolute contra-indication as is bacterial endocarditis. 
Gross regurgitation with considerable enlargement of the left auricle and ventricle renders the operation unlikely to be successful and patients with this state of affairs have a high mortality if subjected to it. $A$ history of embolism makes the operation more hazardous but is not a contraindication. Aortic valvular disease is at present considered a contra-indication when severe, but functional tricuspid incompetence is not.

Auricular fibrillation or calcification of the valve are no longer looked upon as contra-indications, and the onset of pulmonary oedema once regarded as a bar may, in fact, as Brock has stressed, make the necessity for operation one of acute emergency.

\section{Operation}

As experience with the operation of 'commissurotomy' has been gained it has become less complex, and steps which were originally thought to be essential have been abandoned. There is not universal agreement as to which is the best approach; there are exponents of the anterior and of the antero-lateral incision, but a standard long postero-lateral thoracotomy incision with removal of the fifth rib offers few disadvantages and gives adequate exposure and ease of access to the auricle.

When the chest has been opened the pericardium is incised either in front of or behind the phrenic nerve, then either a clamp may be put across the base of the auricular appendage or, if it is felt that thrombi may be dislodged by this procedure, the appendage is opened and allowed to bleed and so to wash out any present. The clamp is placed in position as soon as possible after this incision. If the auricular appendage is too small or is thrombosed, the valve may be approached through the pulmonary veins. This approach was first used successfully by Thompson (I952) although it had been previously attempted by Harken (1948). The index finger is then insinuated through the incision in the appendage into the auricle as the clamp is released. This procedure seldom causes haemorrhage. The mobility of the mitral valve is assessed; the size and shape of the orifice, the presence or absence of palpable regurgitation are noted and the 'commissures' which may be calcified are felt. The finger is then advanced through the orifice and the valve is split along the ' commissures,' the posteromedial split being attempted first as it is the more difficult of the two. If digital splitting is found to to impossible the finger is removed and one of the many cutting devices is then fitted and introduced so that the splits may be started in the right direction, care being taken not to damage the antero-medial cusp.
When as much mobilization of the valve as possible has been achieved the finger is removed, the clamp is re-applied and the auricular appendage is sewn with an unabsorbable suture. Some surgeons remove the appendage in all cases, but this precludes the possibility of a second attack on the valve which may prove necessary as years. go by.

The post-operative course is usually smooth, pulmonary complications are uncommon, the risk of cerebral and peripheral arterial embolism due to dislodgement of clot at the time of operation is ever present and is the chief cause of death following the operation. This accident is, however, uncommon. Transient or permanent irregularity (usually auricular fibrillation) is not uncommon, but it is not yet clear whether its incidence is reduced by the prophylactic use of quinidine and digitalis in the pre-operative and post-operative periods.

\section{Results of the Operation}

The mortality, although depending in part on surgical skill, is largely governed by the skill in selection of patients for the operation. Hence, on the one hand, many of the criteria of unsuitability have only been learnt by experience, whereas on the other many criteria of inoperability have been discarded as practice has rendered the operation safer.

As with many cardiac operations, the early mortality has been heavy, but as experience has grown the figures have become surprisingly low. Brock (195I) in 48 patients subjected to calvulotomy said that eight had died but there had been no fatality in the last 22. More recently his total mortality for 100 cases was 13, which shows a great improvement on the earlier series and the figure now is almost as low as it could be expected to be.

Bailey (195I) in discussing a larger series of 2 I.4 patients gives a mortality of 12.6 per cent. Smaller series published have given a higher figure (Miller and Longmire, $195^{1-3}$ in 14 , and Lam, 195 $1-5$ in 19 ), but almost invariably the seiection has been at fault. Of the 63 cases under Holmes Sellors' (1952) care there have been no fatalities.

The objective assessment of improvement is difficult. Catheterization studies carried out at Guy's and the Brompton Hospitals both before and after operation show that even a persistent greatly raised pulmonary artery pressure which might be thought to have given rise to irreversible damage in the pulmonary arterioles does not materially affect the outcome.

It is difficult to be certain at the time of operation to what extent a valve has been split, but except in cases where almost no mobilization has 
been possible, it has been our experience that the prediction of the result has often been erroneous; in many cases the improvement was much greater than had been anticipated. Bailey (I95I) states that of his 214 cases 41.6 per cent. were excellent, 32.7 per cent. improved and 13 per cent. unaltered after operation. Brock et al. (1952) after a careful follow up of their first 50 cases state that of the 42 survivors, the results were-excellent in 17; good in 15 , four were fair only and five were poor.

Our experience is closely similar to these two series and although there is necessarily a personal factor in assessing these results, which accounts for small differences, there is no doubt that the operation is of enormous value to many of these patients, who from chronic invalidism may be returned to an almost completely normal life.

BIBLIOGRAPHY

F. D'ALLAINS and C. DUBOST (195I), f. Chir., Paris, 67, 389.
BAILEY, C. P. (1950), Four. Thor. Surg., 20, 516.

BAILEY, C. P. (1950), Ibid., 19, 16.

BAILEY, C. P. (1949), Dis. Chest., 15, 4.


(1952), Brit. med. Four., May 17, 1043. BLAND, E. F., and SWEET, R. H. (1949), F.A.M.A., 140 (16),
1259.

BOURNE, G. (1952), Brit. med. F., April, 896.

BROCK, R. C. (1951), Proc. Roy. Soc. Med., 44995.

BROCK, R. C. (1950), Brit. med. F., i, 1283.

BRUNTON, L. (1902), Lancet, i, 352.

BUTTERWORTH, R. F. (1951), four. Thor. Surg., 22, 3 19.

CUTLER, E. C., and LEVINE, S. A. (1923), Boston M. \&' S.F., I88, 1023 .

HARKEN, D. E. (1948), New Eng. Med. Four., 239, 80 r.

HARKEN, D. E. (1950), Four. Thor. Surg., 19, 1.

HARKEN, D. E., DEXTER, L., ELLIS, L. B., FARRAND, R. E., and DICKSON, J. F. (I95I), Ann. Surg., 134, 722.

LAM, C. R. (1951), Arch. Surg., 63, 349.

MILLER, W. H., and LONGMIRE (195I), Surgery, 30, 29.

MURRAY, G. (1950), Arch. Surg., 61, 903.

SELLORS, T. H. (1951), Personal communication.

SOMERVILLE, W. (1952), Personal communication.

SOUTAR, P. W. (1925), Brit. med. F., 2, 603.

'THOMPSON', V. C. (1952), Personal communication.

\title{
RHEUMATISM : THE PRESENT POSITION
}

\author{
By F. Dudley HaRT, M.D., F.R.C.P. \\ Physician-in-Charge, Rheumatism Unit, Westminster Hospital, S.W.I
}

Not many years ago little interest was taken in the articular diseases. The field of ' rheumatism' was a loosely defined one and in this ill-defined area physician, orthopaedic surgeon, physiotherapist and others wandered rather at random. Therapy was and still is largely palliative and empirical. The rheumatoid in particular derived little help from any form of therapy except certain forms of physiotherapy, splinting, support and graded rest, measures which are still the backbone of treatment. Knowledge of the exact aetiological agents, without which therapy must continue to be empirical, is still lacking. Since the war more attention has been devoted to the study of this group of disorders and, when in 1949 Hench and his colleagues reported the dramatic effect of cortisone on rheumatoid arthritis, a great impetus was given to research into study of diseases of the locomotor system. Now, three years later, it is perhaps time to take stock of the present position.

\section{Nomenclature}

A glance at the history of medicine shows certain concertina-like features in the study of disease groups in which the aetiology is unknown. 'A,' a great man in his day, classifies all the diseases and points out their differences, splitting them into sub-groups on clinical and pathological grounds. ' $\mathrm{B}$,' equally eminent, some years later draws attention to points of similarity throughout the whole group and unifies them as best he can. Later ' C,' a brilliant young worker, splits them up again and adds his name to two syndromes which emerge from the reshuffle. In the absence of exact knowledge this can go on indefinitely. A prolonged search for infective agents has, except in a few instances, proved fruitless in the field of rheumatology and there seems little to hope for in this direction with current methods. The pendulum is now over to host reaction rather than invading organism, to disease of soil rather than seed. The recent paper of Kellgren (1952) should be read in this context.

In this country our classification of the rheumatic disorders is in agreement with that used in the United States of America with only few exceptions. Only the more common dișorders are discussed below. 